\title{
Hepatectomía abierta versus laparoscópica dentro de un programa de ERAS (Enhanced Recovery After Surgery). ¿Hay diferencias?
}

\author{
Open versus laparoscopic hepatectomies within an ERAS (Enhanced Recovery After \\ Surgery) program. Are there differences?
}

\author{
Gustavo A. Nari'*, Ernesto Castro-Gutiérrez², José Layún ${ }^{1}$, Laia Falgueras-Verdaguer ${ }^{2}$, Daniela Mariot ${ }^{1}$, \\ María T. Albiol-Quer ${ }^{2}$ y Margarida Casellas-Robert ${ }^{2}$ \\ 'Servicio de Cirugía General, Hospital Tránsito Cáceres de Allende, Córdoba, Argentina; ${ }^{2}$ Sección de Cirugía Hepato-Bilio-Pancreática, Servicio \\ de Cirugía, Hospital Josep Trueta, Gerona, España
}

\begin{abstract}
Resumen
Antecedentes: Los procedimientos laparoscópicos ganaron popularidad porque favorecen una recuperación rápida. El establecimiento de programas ERAS (Enhanced Recovery After Surgery) en cirugía mayor abdominal ha acortado la estancia hospitalaria independientemente de la vía de abordaje. Varios trabajos informan los resultados sobre programas ERAS aplicados a hepatectomías abiertas o laparoscópicas, o comparando una de ellas con el abordaje clásico, pero pocos han comparado los resultados entre ambas dentro de un programa ERAS. Objetivo: Comparar, dentro un mismo programa ERAS, los resultados entre hepatectomías abiertas y laparoscópicas. Método: Se incorporaron a este estudio 36 pacientes sometidos a hepatectomías que fueron abordados por vía laparoscópica o abierta dentro de un programa ERAS. Los objetivos primarios fueron la estancia hospitalaria y el número de complicaciones. Resultados: No hubo diferencias significativas entre ambos grupos en cuanto a estancia hospitalaria ni número de complicaciones. Las diferencias radicaron en un menor tiempo de cirugía y una deambulación más precoz a favor de la cirugía abierta, y una recuperación total mejor a favor del abordaje laparoscópico. Conclusiones: Los datos iniciales sugieren que no habría beneficios en cuanto a estancia hospitalaria e índice de complicaciones de las hepatectomías laparoscópicas sobre las abiertas dentro de un programa ERAS.
\end{abstract}

Palabras Clave: Hepatectomía abierta. Hepatectomía laparoscópica. Programa ERAS.

\begin{abstract}
Background: Laparoscopic procedures have gained popularity because they favor a faster recovery. In the same way, the establishment of an enhanced recovery after surgery (ERAS) program in major abdominal surgery has shortened the hospital stay. There are several studies that report the results on ERAS programs applied to open or laparoscopic hepatectomies or comparing one of them with the classic approach but few have compared the results between both within an ERAS program. Objective: To compare the results between open and laparoscopic hepatectomies in the same ERAS program. Method: Thirty-six patients undergoing hepatectomies were enrolled in this study and were either laparoscopically or open in an identical ERAS program. Hospital stay and the number of complications were taken as primary end points. Results: There were no significant differences between both groups in terms of hospital stay or number of complications. The differences were
\end{abstract}

Correspondencia:

*Gustavo A. Nari

Buchardo, 1200

Fecha de recepción: 15-12-2018

Cir Cir. 2020;88(1):49-55

C.P. 5000, Córdoba, Argentina

Fecha de aceptación: 27-08-2019

DOI: $10.24875 / C I R U .19000972$

Contents available at PubMed

E-mail: gusnari@ hotmail.com

www.cirugiaycirujanos.com

0009-7411/@ 2019 Academia Mexicana de Cirugía. Publicado por Permanyer. Éste es un artículo open access bajo la licencia CC BY-NC-ND (http://creativecommons.org/licenses/by-nc-nd/4.0/). 
in a shorter time of surgery and an earlier ambulation in favor of open surgery and a better full recovery in favor of the laparoscopic approach. Conclusions: Initial data suggest that there would be no benefits in terms of hospital stay and complication rate of laparoscopic hepatectomies over open ones within an ERAS program.

Key Words: Open hepatectomies. Laparoscopic hepatectomies. ERAS program.

\section{Introducción}

Desde la introducción de los programas de recuperación acelerada en cirugía colónica por Kehlet y Wilmore ${ }^{1}$, y la posterior aplicación de los mismos a otras cirugías tales como pancreatectomías, gastrectomías, etc., la utilización de estos programas ha sufrido un efecto multiplicador ${ }^{2-7}$.

Una disminución de la estancia hospitalaria, un menor número de complicaciones y una disminución de los costos son, según diferentes autores, las ventajas más importantes de un programa ERAS (Enhanced Recovery After Surgery) $)^{8-12}$.

Las resecciones hepáticas no quedaron fuera de esta tendencia y, si bien existen varios estudios que miden la aplicación de programas ERAS a las hepatectomías, algunos protocolos presentan diferencias respecto de las variables medidas para valorar su efectividad ${ }^{13}$.

La mayoría de los autores coinciden en destacar una disminución en los tiempos de hospitalización, y por ende en los costos hospitalarios, con un ahorro que rondaría los 2,000 dólares cuando se compara con el manejo perioperatorio habitual ${ }^{14}$. Una disminución del porcentaje de complicaciones es otra de las ventajas aducidas, aunque la literatura es bastante contradictoria en este punto ${ }^{15}$. En un trabajo realizado con anterioridad en el que se compararon hepatectomías abiertas por metástasis colorrectales con manejo tradicional versus ERAS se comprobó una disminución de un $50 \%$ en la estancia hospitalaria, pero no hubo diferencias respecto al índice de complicaciones ni los otros parámetros medidos, aunque esta era una experiencia inicial' ${ }^{16}$.

El abordaje mínimamente invasivo suele ser mencionado como uno de los puntos fuertes para el éxito de un programa ERAS en cirugía mayor abdominal14,17. Existen pocas publicaciones que comparen las resecciones hepáticas abiertas versus las laparoscópicas dentro de un programa ERAS.

El objetivo de esta comunicación es comparar los resultados entre las hepatectomías abiertas y laparoscópicas dentro de un programa ERAS idéntico en dos instituciones, tomando como objetivos primarios la estancia hospitalaria y el número de complicaciones, y como objetivos secundarios la adherencia al protocolo, las pérdidas hemáticas, el inicio de la deambulación y la recuperación funcional al momento del alta.

\section{Método}

Estudio transversal, observacional y descriptivo, sobre la aplicación de un programa ERAS en hepatectomías abiertas versus laparoscópicas llevado a cabo en dos instituciones. Una de ellas (Hospital Josep Trueta, Gerona, España) fue la proveedora de los casos operados por laparoscopía, mientras que la otra (Hospital Tránsito Cáceres de Allende, Córdoba, Argentina) aportó la mayoría de las hepatectomías realizadas a cielo abierto. Las cirugías laparoscópicas que necesitaron conversión a cirugía abierta fueron incluidas dentro de las hepatectomías abiertas en el análisis.

Un programa ERAS con puntos básicos en el preoperatorio, el intraoperatorio y el posoperatorio fue consensuado por ambos equipos y es el que se expone a continuación.

\section{Programa ERAS aplicado}

1) Preoperatorio:

- Información completa del procedimiento, alcances del mismo e involucrar al paciente en su desarrollo.

- Día preoperatorio: dieta (la noche anterior a la cirugía). Ingesta de líquidos ricos en hidratos de carbono hasta 2 horas previas a la cirugía.

- Uso de heparina de bajo peso molecular 12 horas antes de la cirugía

2) Intraoperatorio:

- Control de fluidoterapia (balance cerca de cero).

- Drenajes (preferentemente no).

- Sonda nasogástrica (retirada al finalizar la cirugía).

- Catéter epidural o morfina intratecal. 
3) Posoperatorio:

- Retirada de la fluidoterapia dentro de las primeras 48 horas.

- Indicación reglada de procinéticos.

- Inicio de dieta líquida dentro de las primeras 24 horas.

- Inicio de dieta regular dentro de las primeras 48 horas.

- Retirada de sonda vesical dentro de las primeras 24 horas.

- Inicio temprano de la deambulación.

Los criterios utilizados para considerar una recuperación funcional total fueron:

- Control del dolor con analgésicos orales.

- No utilización de fluidos intravenosos.

- Movilización al nivel preoperatorio.

- Ingesta de alimentos sólidos.

- Bilirrubina sérica normal o con descenso hacia la normalidad.

La recuperación funcional fue valorada en el momento del alta del paciente. La adherencia al programa ERAS se valoró a través de un listado de verificación impreso en el que el cirujano marcaba «sí» 0 «no» en el cumplimiento de cada uno de los ítems de la lista.

Se evaluaron los datos demográficos, los referentes a la etiología y las hepatectomías, el curso posoperatorio y aquellos referidos al programa ERAS. Las hepatectomías fueron registradas según la clasificación de Brisbane ${ }^{18}$, las fístulas biliares según la propuesta del International Study Group of Liver Surgery $(\text { ISGLS })^{19}$, la insuficiencia hepática según el criterio del $50 / 50 \%{ }^{20}$ y las complicaciones según la clasificación de Dindo-Clavien ${ }^{21}$. Todos los pacientes fueron operados con disminución de la Presión Venosa Central (PVC) controlada, y en todos se midió la presión arterial media. En todos los pacientes se utilizó profilaxis antitrombótica con heparina de bajo peso molecular asociada a vendaje de miembros inferiores, así como profilaxis antibiótica. En todos los pacientes se realizó ecografía intraoperatoria.

\section{Análisis estadístico}

Las variables categóricas se describen mediante proporciones, las variables continuas simétricas mediante el promedio y el desvío estándar, y las variables continuas asimétricas a través de la mediana y el rango.

Se utilizó la prueba de ji al cuadrado para la comparación de proporciones entre grupos, y el test exacto de Fisher cuando correspondía. Para las
Tabla 1. Datos demográficos

\begin{tabular}{lccc}
\hline Variable & $\begin{array}{c}\text { Cirugía abierta } \\
(\mathbf{n}=\mathbf{2 1})\end{array}$ & $\begin{array}{c}\text { Cirugía } \\
\text { laparoscópica } \\
(\mathbf{n}=\mathbf{1 5})\end{array}$ & $\mathbf{p}$ \\
\hline Sexo masculino & $66.7 \%$ & $93.3 \%$ & 0.104 \\
Edad promedio \pm DE (años) & $58.1 \pm 11.1$ & $59.9 \pm 13.5$ & 0.810 \\
ASA 2 & $23.8 \%$ & $13.3 \%$ & 0.674 \\
ASA 3 & $76.2 \%$ & $86.7 \%$ & \\
Consumo de alcohol & $4.8 \%$ & $6.7 \%$ & 1.000 \\
Tabaco & $33.3 \%$ & $26.7 \%$ & 0.729 \\
IMC (kg/m²) & 26.9 & 27.3 & 0.961 \\
Ejercicio físico & $28.6 \%$ & $13.3 \%$ & 0.424 \\
\hline DE: desvío estándar; IMC: índice de masa corporal. & &
\end{tabular}

comparaciones de promedios entre grupos se utilizó el test de Mann-Whitney, que se consideró más apropiado que el test $t$ de Student debido al tamaño muestral reducido. En todos los casos se consideró como significativo un error alfa bilateral $<5 \%$.

Los datos se analizaron con el software PASW 18.

\section{Resultados}

Se incorporaron al estudio 36 pacientes, de los cuales 20 inicialmente fueron abordados de forma abierta y 16 por vía laparoscópica, pero uno de ellos fue convertido $(6.25 \%)$ e incorporado dentro del grupo de las hepatectomías abiertas, quedando la distribución definitiva con 21 pacientes en el grupo de abordaje abierto y 15 en el de abordaje mínimamente invasivo.

En la tabla 1 se muestran los datos demográficos de los pacientes. No hubo diferencias en lo que respecta a distribución por sexo, edad, riesgo operatorio, índice de masa corporal, etc.

Las indicaciones de las hepatectomías fueron en su mayoría por metástasis de origen colorrectal (15 en el grupo de abiertas y 10 en el de laparoscópicas), y la mayoría fueron resecciones limitadas. En la tabla 2 pueden verse los resultados referentes a las hepatectomías; en lo que respecta al tiempo operatorio, hubo una diferencia estadísticamente significativa a favor de la técnica abierta. Otra variable en la que, si bien no se obtuvo diferencia significativa, pero hubo un importante número de casos en la técnica abierta, fueron las resecciones simultáneas: nueve (42.8\%) frente a uno $(6.6 \%)$.

Por otra parte, en los pacientes sometidos a cirugía abierta se utilizó en mayor medida la colocación de 
Cirugía y Cirujanos. 2020;88(1)

Tabla 2. Datos de las hepatectomías

\begin{tabular}{lccc}
\hline Variable & Cirugía abierta $\mathbf{( n = 2 1 )}$ & Cirugía laparoscópica $(\mathbf{n}=\mathbf{1 5})$ & $\mathbf{p}$ \\
\hline Hepatectomía mayor & $1(4.76 \%)$ & $2(13.3 \%)$ & 0.559 \\
Resección simultánea & $9(42.8 \%)$ & $1(6.6 \%)$ & 0.054 \\
Tiempo operatorio promedio $(\mathrm{min})$ & 150 & 297.5 & 0.001 \\
Pérdidas hemáticas $>300 \mathrm{ml}$ & $42.9 \%$ & $46.7 \%$ & 0.821 \\
Complicaciones & $3(14.3 \%)$ & $2(13.3 \%)$ & 1.000 \\
Colocación de drenajes & $6(28.7 \%)$ & $1(6.6 \%)$ & 0.200 \\
Estancia hospitalaria sin reingreso (promedio, días) & 3.86 & 3.93 & 0.696 \\
Estancia hospitalaria con reingreso (promedio, días) & 4.10 & 5.00 & 0.841 \\
\hline
\end{tabular}

Tabla 3. Datos del programa ERAS

\begin{tabular}{lccc}
\hline Variable & $\begin{array}{c}\text { Cirugía } \\
\text { abierta } \\
(\mathbf{n}=\mathbf{2 1})\end{array}$ & $\begin{array}{c}\text { Cirugía } \\
\text { laparoscópica } \\
(\mathbf{n}=\mathbf{1 5})\end{array}$ & $\mathbf{p}$ \\
\hline Adherencia 100\% & $13(61.9 \%)$ & $9(60 \%)$ & 1.000 \\
Inicio de deambulación & $12.79 \mathrm{~h}$ & $26.5 \mathrm{~h}$ & 0.000 \\
Tiempo retirada sonda vesical & $25.7 \mathrm{~h}$ & $29.6 \mathrm{~h}$ & 0.120 \\
Tiempo retirada drenaje & $30 \mathrm{~h}$ & $120 \mathrm{~h}$ & 0.114 \\
Tiempo retirada fluidoterapia & $35.3 \mathrm{~h}$ & $34.53 \mathrm{~h}$ & 0.935 \\
Recuperación funcional & $13(61.9 \%)$ & $15(100 \%)$ & 0.011 \\
Reingreso hospitalario & $2(9.5 \%)$ & $2(13.3 \%)$ & 1.000 \\
\hline
\end{tabular}

drenajes (6 vs. 1). La estancia hospitalaria con y $\sin$ reingreso no presentó diferencias entre ambos grupos, al igual que las complicaciones (3 vs. 2; 14.3 vs. 13.3\%); los pacientes sometidos a cirugía abierta tuvieron complicaciones tipo I, mientras que los sometidos a la vía laparoscópica tuvieron complicaciones más graves (tipo III). No hubo mortalidad.

En la tabla 3 pueden observarse los resultados de la aplicación del programa ERAS, donde los datos sobresalientes son una deambulación más rápida en los pacientes sometidos a cirugía abierta (12.8 vs. $26.5 \mathrm{~h} ; \mathrm{p}=0.000$ ) y una recuperación funcional mayor a favor de la vía laparoscópica (61.9 vs. $100 \%$; $p=0.011)$. En el resto de las variables medidas no hubo diferencias significativas, aunque la duración de la instalación del drenaje fue mucho más corta en la cirugía abierta (30 vs. $120 \mathrm{~h}$ ).

\section{Discusión}

La aplicación de un programas ERAS en cirugía mayor de colon y recto ha mostrado beneficios para los pacientes y ha favorecido su aplicación a otros órganos. El uso de programas ERAS en cirugía hepática se viene implementando de manera creciente, observándose en la mayoría de los trabajos de manera consistente una disminución de la estancia hospitalaria y, por ende, de los costes 22,23 .

Hay pocos estudios en los que se compare la aplicación de programas ERAS entre hepatectomías abiertas y laparoscópicas. La vía de abordaje mínimamente invasiva ha sido considerada como uno de los puntos fuertes para lograr una recuperación más rápida y de mayor calidad, comparada con la cirugía abierta, en los pacientes sometidos a cirugía hepática ${ }^{1724-29}$. Los estudios refieren una franca mejoría en cuanto a estancia hospitalaria, disminución de las complicaciones y menor pérdida hemática, con la consiguiente disminución de las necesidades transfusionales, en comparación con la técnica abierta. También manifiestan que hay una disminución en el tiempo de íleo posoperatorio. Croome y Yamashita ${ }^{28}$ concluyen que los pacientes operados por vía laparoscópica inician de manera más temprana la ingestión de alimentos y requieren menor analgesia intravenosa. Estos estudios fueron realizados fuera de protocolos ERAS $^{24-29}$ y nuestro estudio apunta a responder si existen diferencias entre ambas técnicas dentro de un programa ERAS.

Para este estudio se consensuó un programa entre las dos instituciones teniendo en cuenta algunos de los puntos con mayor nivel de evidencia y recomendación sugeridos por la ERAS Society ${ }^{17}$.

De la comparación de ambos grupos y atendiendo a los objetivos del trabajo notamos que la estancia hospitalaria con y sin reingresos fue similar. La estancia hospitalaria sin reingresos fue de 3.86 días para los pacientes operados con técnica abierta y de 3.93 
días para el abordaje laparoscópico ( $p=0.696$ ), mientras que cuando se sumaron los días de reingreso la estancia hospitalaria fue de 4.10 y 5.00 días, respectivamente $(p=0.841)$. La estancia hospitalaria reportada en la literatura oscila entre 3 y 7 días $^{30-34}$. En todas las series se reporta una disminución significativa de varios días en comparación con el manejo convencional, lo que tiene un impacto directo en los costes de este tipo de cirugía ${ }^{14}$.

En lo que se refiere a las complicaciones, tampoco hubo diferencias significativas entre ambos grupos $(p=1.000)$. El porcentaje de complicaciones fue del $14.3 \%$ para las hepatectomías abiertas y del $13,3 \%$ para las laparoscópicas. Las complicaciones del grupo de cirugía abierta fueron de menor gravedad que las del grupo laparoscópico (Dindo-Clavien tipo I vs. tipo III, respectivamente), que requirieron procedimientos mínimamente invasivos para su resolución. El porcentaje referido en la literatura es más elevado y ronda el $30 \%$ en la mayoría de las series $^{32,33}$. Por otra parte, Stoot, et al. ${ }^{34}$ reportan solo dos complicaciones en 13 hepatectomías laparoscópicas (15.4\%). Es probable que la justificación de nuestro bajo porcentaje de complicaciones radique principalmente en que el número de hepatectomías mayores en ambos grupos es pequeño (abierta $4.76 \%$ y laparoscópica $13.3 \%$ ), lo que coincidiría con algunos autores que han notado diferencias en cuanto al número de complicaciones y días de estancia cuando se compararon hepatectomías mayores y menores ${ }^{31}$.

La adherencia a la totalidad del protocolo se logró en el $61.9 \%$ de los pacientes con cirugía abierta y en el $60 \%$ de los pacientes con cirugía laparoscópica $(p=1.000)$. Las principales dificultades en su cumplimiento se vieron principalmente en el posoperatorio, en el inicio de la deambulación en los pacientes sometidos a cirugía laparoscópica (26.5 h), mientras que en cirugía abierta el inicio de la deambulación fue más temprano (media de $12.79 \mathrm{~h} ; \mathrm{p}=0.000$ ). Esta diferencia podría explicarse por dos motivos: uno, que tal vez la utilización del neumoperitoneo sea un factor a tener en cuenta, y el otro, que probablemente se encuentre justificado en que la duración del acto operatorio fue más prolongada en la cirugía laparoscópica que en la abierta (297 vs. $150 \mathrm{~min} ; \mathrm{p}=0.001$ ). Por otra parte, coincidimos con algunos autores en que habrá mayor adherencia a los protocolos cuanto más completa y mejor comprendida sea la información inicial recibida por el paciente, y mayor sea el compromiso que este asuma ${ }^{35}$.
Algunos autores ${ }^{36}$ reportan que en las primeras 24 horas indican que el paciente permanezca sentado en la cama o en una silla, y pasado este tiempo inicien la deambulación. Esta es una decisión que se toma de manera individual de acuerdo con la percepción del cirujano y de las características del enfermo.

En lo que respecta a las pérdidas hemáticas, no hubo diferencias significativas ( $p=0.821$ ) entre los dos grupos; en ambos, más del $50 \%$ tuvieron pérdidas menores de $300 \mathrm{ml}$. Algunos autores ${ }^{34}$ reportan que la cirugía laparoscópica disminuye de manera significativa las pérdidas hemáticas en comparación con los cuidados tradicionales.

La recuperación funcional total al momento del alta presentó diferencias a favor de la cirugía laparoscópica $(p=0,011)$ : la lograron 13 pacientes $(61.9 \%)$ del grupo de cirugía abierta y $15(100 \%)$ del grupo de cirugía laparoscópica. La diferencia en este factor radicó en que, en el grupo de cirugía abierta, el control del dolor posoperatorio al momento del alta fue menos satisfactorio. Es probable que, así como la realización del neumoperitoneo y el mayor tiempo quirúrgico demoraron la deambulación en el grupo de pacientes operados por laparoscopia, su característica mínimamente invasiva haya favorecido un mejor control de dolor al momento del alta. Otro punto importante que podría justificar esta diferencia es que en el grupo de pacientes operados de manera laparotómica hubo nueve que recibieron de manera simultánea una resección colónica, mientras que en el grupo con abordaje laparoscópico tan solo en uno se asoció una resección simultánea $(p=0,054)$. Wong Lun Hing, et al..$^{30}$ refieren que la mayoría de sus pacientes lograron la recuperación funcional total en el quinto día de posoperatorio. La diferencia de casi 1 día en la estancia hospitalaria entre la cirugía abierta y la laparoscópica de nuestra serie podría ser también otra forma de entender las diferencias entre ambos grupos en este punto.

En lo que respecta a otros parámetros comparados, en la cirugía abierta se colocaron más drenajes que en la laparoscópica (6-28.5 vs. 1-6.6\%); la elección de la colocación de drenajes intraabdominales quedó a consideración del cirujano. Es importante destacar nuevamente que, por un lado, en el grupo de cirugía abierta hubo un mayor número de resecciones simultáneas, y por el otro, que en el grupo de cirugía laparoscópica las complicaciones pertenecieron al tipo III de Dindo-Claven y consistieron en colecciones intraabdominales que requirieron la colocación de drenajes percutáneos. Algunos autores reportan un 20\% 
de colocación de drenajes con la técnica abierta ${ }^{13}$. Coincidimos con la mayoría de los autores en que en un gran número de pacientes la colocación de drenajes en las hepatectomías es innecesario ${ }^{37}$; tal vez sería conveniente protocolizar en qué pacientes deben dejarse drenajes. Algunos autores hallaron que los pacientes con hepatectomías con una duración superior a 350 minutos, con una pérdida hemática superior a $650 \mathrm{ml}$ o con fuga biliar durante la cirugía son tributarios de colocación de drenajes ${ }^{38}$.

\section{Conclusiones}

Como conclusión podemos decir que en nuestra serie de pacientes no hubo diferencias en cuanto a días de estancia ni número de complicaciones; que efectivamente hay una reducción de los días de internación cuando se compara con el manejo tradicional; que nuestro estudio es una muestra pequeña y que se requiere un mayor número de pacientes; que el número de hepatectomías mayores es escaso y que, en la medida en que incorporemos más, los resultados pueden variar; y finalmente, que la información inicial al paciente es fundamental para poder llevar adelante un programa ERAS en cualquier unidad de cirugía hepática.

\section{Conflicto de intereses}

Los autores no poseen ningún conflicto de intereses ni han recibido financiamiento para la realización del presente trabajo.

\section{Responsabilidades éticas}

Protección de personas y animales. Los autores declaran que los procedimientos seguidos se conformaron a las normas éticas del comité de experimentación humana responsable y de acuerdo con la Asociación Médica Mundial y la Declaración de Helsinki.

Confidencialidad de los datos. Los autores declaran que han seguido los protocolos de su centro de trabajo sobre la publicación de datos de pacientes.

Derecho a la privacidad y consentimiento informado. Los autores declaran que en este artículo no aparecen datos de pacientes.

\section{Bibliografía}

1. Kehlet $\mathrm{H}$, Wilmore D. Multimodal strategies to improve surgical outcome Am J Surg. 2002;183:630-41.

2. Gouvas N, Tan E, Windsor A, Xynos E, Tekkis P. Fast-track vs standard care in colorectal surgery: a meta-analysis update. Int $\mathrm{J}$ Colorectal Dis. 2009;24:1119-31.
3. Spanjersberg W, Reurings J, Keus F, van Laarhoven C. Fast track surgery versus conventional recovery strategies for colorectal surgery. Cochrane Database Syst Rev. 2011;(2):CD007635.

4. Dai J, Jiang Y, Fu D. Reducing postoperative complications and improving clinical outcome: enhanced recovery after surgery in pancreaticoduodenectomy. A retrospective cohort study. Int J Surg. 2017;39:176-81.

5. Bai X, Zhang X, Lu F, Li G, Gao S, Lou J, et al. The implementation of an enhanced recovery after surgery (ERAS) program following pancreatic surgery in an academic medical center of China. Pancreatology. 2016;16:665-70

6. Tanaka R, Lee S, Kawai M, Tashiro K, Kawashima S, Kagota S, et al. Protocol for enhanced recovery after surgery improves short-term outcomes for patients with gastric cancer: a randomized clinical trial. Gastric Cancer. 2017;20:861-71.

7. Abdikarim I, Cao X, Li S, Zhao Y, Taupik Y, Wang Q. Enhanced recovery after surgery with laparoscopic radical gastrectomy for stomach carcinomas. World J Gastroenterol. 2015;21:1339-44.

8. Greco M, Capretti G, Beretta L, Gemma M, Pecorelli N, Braga M. Enhanced recovery program in colorectal surgery: a meta-analysis of randomized controlled trials. World J Surg. 2014;38:1531-41.

9. Muller S, Zalunardo M, Hubner M, Clavien P, Demartines N, Zurich fast track Study Group. A fast track program reduces complications and length of hospital stay after open colonic surgery. Gastroenterology. 2009;136:942-7.

10. Roulin D, Donadini A, Gander S, Griesser A, Blanc C, Hubner M, et al. Cost-effectiveness of the implementation of an enhanced recovery protocol for colorectal surgery. Br J Surg. 2014;100:1108-14.

11. Ni C, Yang $\mathrm{Y}$, Chang $\mathrm{Y}, \mathrm{Cai} \mathrm{H}, \mathrm{Xu} \mathrm{B}$, Yang $\mathrm{F}$, et al. Fast track surgery improves postoperative recovery in patients undergoing partial hepatectomy for primary liver cáncer: a prospective randomized conrolled trial. Eur J Surg Oncol. 2013;39:542-7.

12. Jones C, Kelliher L, Dickinson M, Riga A, Worthinton T, Scott M, et al. Randomized clinical trial on enhanced recovery versus standard care following open liver resection. Br J Surg. 2013;100:1015-24.

13. Connor S, Cross A, Sakowska M, Linscott D, Woods J. Effects of introducing an enhanced recovery after surgery programme for patients undergoing open hepatic resection. HPB. 2013;15:294-301.

14. Liang $\mathrm{X}$, Ying $\mathrm{H}$, Wang $\mathrm{H}, \mathrm{Xu} \mathrm{H}, \mathrm{Yu} \mathrm{H}, \mathrm{Cai} \mathrm{L}$, et al. Enhanced recovery program versus traditional care in laparoscopic hepatectomy. Medicine. 2016;95:e2835

15. Dasari B, Rahman R, Shakeeb K, Bennett D, Hodson J, Isaac J, et al. Safety and feasibility of an enhanced recovery pathway after a liver resection: prospective cohort study. HPB. 2015;17:700-6.

16. Nari G, Molina L, Gil F, Viotto L, Layún J, Mariot D, et al. Enhanced Recovery After Surgery (ERAS) in open liver resections for hepatic colorectal metastasis. Initial experience. Rev Argent Cirug. 2016;108:9-13.

17. Melloul E, Hubner M, Scott M, Snowden C, Prentis J, Dejong C, et al. Guidelines for perioperative care for liver surgery: Enhanced Recovery After Surgery (ERAS) Society recommendations. World J Surg. 2016;40:2425-40.

18. Strasberg SM, Phillips C. Use and dissemination of the Brisbane 2000 nomenclature of liver anatomy and resections. Ann Surg. 2013;257:377-82.

19. Koch M, Garden J, Padbury R, Rahbari N, Adam R, Capussotti L, et al Bile leakage after hepatobiliary and pancreatic surgery: a definition and grading of severity by the International study group of liver surgery. Surgery. 2011;149:680-8.

20. Balzan S, Belghitti J, Farges O, Ogata S, Sauvanet A, Delafosse D, et al. The 50/50 criteria on postoperative day 5. Ann Surg. 2005:242:824-9.

21. Dindo D, Demartines N, Clavien PA. Classification of surgical complications. A new proposal with evaluation in a cohort of 6336 patients and results of a survey. Ann Surg. 2004;240:205-13.

22. Yang R, Tao W, Chen Y, Zhang B, Tang J, Zhong S, et al. Enhanced recovery after surgery programs versus traditional perioperative care in laparoscopic hepatectomy: a meta-analysis. Int J Surg. 2016;36:274-82.

23. Page A, Ejaz A, Spolverato G, Zavadsky T, Grant M, Galante D, et al Enhanced recovery after surgery protocols for open hepatectomy physiology, immunomodulation and implementation. J Gastrointest Surg. 2015:19:387-99.

24. Rao A, Ahmed I. Laparoscopic versus open liver resection for benign and malignant hepatic lesions in adult. Cochrane Database Syst Rev. 2013;(5):CD1016.

25. Parks K, Kuo Y, Davis J, O’Brien B, Hagopian E. Laparoscopic versus open liver resection: a meta-analysis of long term outcome. HPB. 2014:16:109-18.

26. Nguyen K, Marsh J, Tsung A, Steel M, Gamblin T, Geller D. Comparative benefits of laparoscopic vs open hepatic resection: a critical appraisal. Arch Surg. 2011;146:348-56.

27. Mirnezami R, Mirnezami H, Chandrakumaran K, Abu Hilal M, Pearce N, Primrose J, et al. Short and long-term outcomes after laparoscopic and open hepatic resection: systematic review and meta-analysis. HPB. 2011;13:295-308

28. Croome K, Yamashita M. Laparoscopic versus open hepatic resection for benign and malignant tumors: an updated and meta-analysis. Arch Surg. 2010;145:1109-18 
29. Simillis C, Constantinides V, Tekkis P, Darzi A, Lovegrove R, Jiao L, et al. Laparoscopic versus open hepatic resections for benign and malignant neoplasms - a meta-analysis. Surgery. 2007;141: 203-11.

30. Wong-Lun-Hing E, van Dam R, van Breukelen G, Tanis P, Ratti F, van Hillegersberg R, et al. Randomized clinical trial of open vs laparoscopic left lateral hepatic sectionectomy within an enhanced recovery after surgery programme. Br J Surg. 2017;104:525-35

31. Yip V, Dunne D, Samuels S, Tan C, Lacasia C, Tang J, et al. Adherence to early mobilization: key for successful enhanced recovery after liver resection. Eur J Surg Oncol. 2016;42:1561-7.

32. Dunne D, Yip V, Jones R, McChesney E, Lythgoe D, Psarelli E, et al. Enhanced recovery in the resection of colorectal liver metastases. J Surg Oncol. 2014;110:197-202.

33. Van Dam R, Hendry P, Coolsen M, Bemelmans M, Lassen K, Revhaug A et al. Initial experience with a multimodal enhanced recovery after surgery programme in patients undergoing liver resection. $\mathrm{Br} J$ Surg. 2008;95:969-75.
34. Stoot J, van Dam R, Busch O, van Hillegersberg R, De Boer M, Olde Damnik S, et al. The effect of multimodal fats-track programme on outcomes in laparoscopic liver surgery: a multicentre pilot study. HPB. 2009;11:140-4.

35. Wong Lun Hing E, van Dam R, Heijnen L, Busch O, Terkivatan T, van Hillegersberg R, et al. Is current perioperative practice in hepatic surgery based on enhanced recovery after surgery (ERAS) principles? World J Surg. 2014;38:1127-40.

36. Lee A, Hung Chiu C, Cho M, Gomersall C, Lee K, Cheung Y, et al. Factors associated with failure of enhanced recovery protocol in patients undergoing major hepatobiliary and pancreatic surgery: a retrospective cohort study. BMJ Open. 2014;4:e005330.

37. Wong Lun Hing E, van Woerden V, Lodewick T, Bermelmans M, Olde Damink S, Dejong C, et al. Abandoning prophylactic abdominal drainage after hepatic surgery: a 10 years of non-drain policy in an enhanced recovery after surgery environment. Dig Surg. 2017;34:411-20.

38. Bekki Y, Yamashita Y, Itoh S, Harimoto N, Shirabe K, Maehara Y. Predictors of effectiveness of prophylactic drain after hepatic resection. World J Surg. 2015;39:2543-9. 